\title{
Kowalewski's Asymptotic Method, Kac-moody Lie Algebras and Regularization
}

\author{
M. Adler ${ }^{\star}, 1$ and P. van Moerbeke ${ }^{\star \star, 2}$ \\ 1 Brandeis University, Waltham, MA 02254, USA \\ 2 University of Louvain, B-1348 Louvain-la-Neuve, Belgium, and Brandeis University, \\ Waltham, MA 02254, USA
}

\begin{abstract}
We use an effective criterion based on the asymptotic analysis of a class of Hamiltonian equations to determine whether they are linearizable on an abelian variety, i.e., solvable by quadrature. The criterion is applied to a system with Hamiltonian$$
H=1 / 2 \sum_{i=1}^{l} p_{i}^{2}+\sum_{i=1}^{l+1} \exp \left(\sum_{j=1}^{l} N_{i j} x_{j}\right),
$$

parametrized by a real matrix $N=\left(N_{i j}\right)$ of full rank. It will be solvable by quadrature if and only if for all $i \neq j, 2\left(N N^{T}\right)_{i j}\left(N N^{T}\right)_{j j}^{-1}$ is a nonpositive integer, i.e., the interactions correspond to the Toda systems for the Kac-Moody Lie algebras. The criterion is also applied to a system of Gross-Neveu.
\end{abstract}

A completely integrable Hamiltonian system in a phase space of dimension $2 l$ possesses $l$ independent commuting integrals. Under a compactness condition, the system executes linear motion on an $l$-dimensional torus defined by these integrals. In most classical cases, the torus is defined by (real) polynomial functions of appropriately chosen phase variables, and the transformation to the separating variables is also algebraic. Moreover, the equations of motion in these new variables are solved by quadrature; it means geometrically that the above torus has an algebraic addition law and that the solutions are straight lines with regard to this law. In most examples, the real torus above is part of a complex torus with algebraic addition law. A Hamiltonian system will be called algebraically completely integrable if it can be linearized on an abelian variety (complex algebraic torus with algebraic addition law).

This paper deals with a criterion for algebraic complete integrability, inspired by work of Kowalewski. In celebrated papers [7,8], she has shown that the only

* $\quad$ Supported in part by NSF contract MCS 79-17385

$\star \star$ Supported in part by NSF contract MCS 79-05576 
algebraically completely integrable systems among the rigid body motions are Euler's rigid body, Lagrange's top and the famous Kowalewski top. Her method is based on the idea that if the system is to be algebraically completely integrable, and if the phase variables of the problem are to be algebraic (abelian) functions, then the phase variables of the problem must be meromorphic in time. In addition, the trajectories which blow up (as they must) are nicely parametrized by a codimension one family of parameters. This implies the existence of enough codimension one parameter families of (complex) pole solutions of the system so that all the (abelian) phase variables get a chance to blow up (not necessarily simultaneously). The sufficiency of this criterion has not been established. We remark that the parameters of the pole solutions play the role of regularizing variables when the equations blow up.

This paper breaks up into two sections. The first section deals with a system governed by exponential non-nearest neighbor interactions, with Hamiltonian

$$
H=\frac{1}{2} \sum_{1}^{l} p_{i}^{2}+\sum_{i=1}^{l+1} \exp \left(\sum_{j=1}^{l} N_{i j} x_{j}\right),
$$

parametrized by a real matrix $N=\left(N_{i j}\right)$ of full rank. It will be algebraically completely integrable if and only if for all $i \neq j, 2\left(N N^{\dagger}\right)_{i j}\left(N N^{\dagger}\right)_{i j}^{-1}$ is a nonpositive integer, i.e., the interactions correspond to the Toda systems for the Euclidean Lie algebras. These systems turn out to be the systems first introduced by Bogoyavlensky [10].

These systems turn out to be the systems first introduced by Bogoyavlensky [10].

In the second section, the criterion is applied to the classical version of the Gross-Neveu type model, as suggested by Shankar [11]. This is a Hamiltonian system with energy of the form

$$
H=\frac{1}{2} \sum_{1}^{l} p_{i}^{2}+\sum \exp \langle\alpha, q\rangle,
$$

where the second sum extends over the root system of a simple Lie algebra $R$. We show that for the case $R=\operatorname{sl}(3)$ and $\operatorname{sl}(4) \simeq \mathrm{O}(6)$, the system fails to be algebraically integrable.

In [12], we apply the criterion to a class of geodesic motions in SO(4). Specifically, we consider a system of differential equations

$$
\dot{X}=[X, \Lambda X] \text { with } X+X^{\dagger}=0,
$$

where $(\Lambda X)_{i j}=\Lambda_{i j} X_{i j}$ with $\Lambda_{i j}=\Lambda_{j i}$. This system is shown to be algebraically completely integrable with $\Lambda_{i j}, 1 \leqq i<j \leqq 4$, all distinct and $X_{i j}$ abelian functions if and only if

$$
\Lambda_{i j}=\frac{\beta_{i}-\beta_{j}}{\alpha_{i}-\alpha_{j}}
$$




\section{About the Complete Integrability of an Exponential System of Differential Equations}

This section deals with a lattice governed by exponential non-nearest neighbor interactions, described in the introduction. As will be explained in Remark 4, the equations of motion can be transformed into the following set of differential equations :

$$
\begin{aligned}
& \dot{x}_{i}=x_{i} \sum_{j=1}^{l+1} e_{i j} u_{j}, \quad 1 \leqq i \leqq l+1, \\
& \dot{u}_{i}=\sum_{j=1}^{l+1} x_{j} e_{j i},
\end{aligned}
$$

where $E \equiv\left(e_{i j}\right)$ is a real square matrix of size $l+1$ and rank $l$, having a null vector with entries all of the same sign. This system defines a Hamiltonian vector field for a symplectic structure to be explained below, with Hamiltonian

$$
H=\frac{1}{2} \sum_{i=1}^{l+1} u_{i}^{2}-\sum_{i=1}^{l+1} x_{i} .
$$

The assumption on the rank of $E$ implies that the system has exactly two extrainvariants leading to zero vector fields. The main point of this section is to show that the Toda equations related to the Euclidean Lie algebras are the only algebraically integrable systems with abelian functions $u_{i}$ and $x_{i}$. They include, in particular, the well known periodic Toda equations related to the simple Lie algebras as discussed in Adler and van Moerbeke $[1,2]$.

The $2(l+1)$-dimensional system (1) has two trivial invariants; it can therefore be reduced to a $2 l$-dimensional system. Let it be linearizable on an $l$-dimensional abelian variety $\mathscr{A}$, for which $x_{i}$ and $u_{i}$ are abelian functions. As a result, the system must have $l$ invariants in involution besides the two trivial ones; one of them, of course, is the energy. Each of these invariants can take on arbitrary values. Therefore, if the initial conditions are not specified, the solution must depend on these $l+2$ invariants.

Besides, since $x_{i}$ and $u_{j}$ are abelian functions on $\mathscr{A}$, they each blow up along a piece of a codimension 1 divisor $\mathscr{D}$ on the variety $\mathscr{A}$ in a meromorphic fashion. Hence, for an open set of initial conditions, the system will blow up in a finite time (possibly complex). This assumes that for a generic set of tori, each irreducible piece of $\mathscr{D}$ cannot be an invariant subset of the flow; this will be shown in Remark 1 below. As a result, the solutions $x_{i}$ and $u_{j}$ of (1) admit Laurent expansions in $t$ near the divisor $\mathscr{D}$; since the initial conditions are not specified, these expansions depend on the $l+2$ parameters mentioned above, and, on the point where the trajectory hits the $l$-1-dimensional divisor $\mathscr{D}$, adding another $l-1$ free parameters.

In general, $u_{i}$ or $x_{j}$ must blow up along one or several irreducible pieces of the divisor $\mathscr{D}$ and the expansions of $u_{i}$ and $x_{j}$ may appear very different along these different pieces. The coefficients of these Laurent expansions are determined by induction: except for the leading one, the coefficients are given, at every step, by a linear system of equations, whose right hand side contains the coefficients 
previously obtained. Therefore only a degeneracy in the first (non-linear) equation or a vanishing of the determinant of the linear systems can be responsible for the $2 l+1$ free parameters in the expansions.

We conclude that if the system is completely integrable in the sense above, Eqs. (1) must have enough distinct expansions such that each $u_{i}$ or $x_{j}$ blows up at least once and such that each expansion depends on $2 l+1$ free parameters. It is needless to say that this requirement is a highly exceptional state of affairs. Among the systems (1), only the matrices $E$ (modulo multiplication on the right with an orthogonal matrix) listed in Appendix 1, or, in other words, the Toda equations corresponding to the Euclidean Lie algebras, enjoy these properties. We also note that any formal pole solution to (1) actually converges. For, since Eq. (1) is a quadratic differential equation, any formal power series solution is a convergent series, as is easily seen by the majorant method (see [5, p. 57]). Thus quadratic differential equations are distinguished by this fact.

Remark 1. The criteria explained above depend upon the fact that the divisor $\Gamma=\left\{u_{i}^{-1}=0\right\}$ cannot, for an open set of tori, be an invariant set of the flow. For if $\Gamma$ is invariant and if $\phi^{s}$ is one of the flows commuting with our given one and transversal to $\Gamma$, then $\phi^{s}(\Gamma) \equiv \Gamma^{s}$ would also be invariant. Since $\Gamma$ is a closed set in our torus, it is compact, as is $\Gamma^{s}$. Note that $\Gamma^{s} \cap \Gamma^{s^{\prime}}$ is empty for $0<s \neq s^{\prime}<\varepsilon, \varepsilon$ small, as the vector field of $\phi^{s}$ is everywhere independent from the original vector field. If a solution starts on $\Gamma^{s}, 0<s<\varepsilon$, it remains on $\Gamma^{s}$ and so, by the compactness of $\Gamma$ and $\Gamma^{s}$, there exists a constant $M_{s}$ such that $\left|u_{i}(t)\right| \leqq M_{s}$ for all complex $t$. But a bounded meromorphic function must be constant and so $u_{i}(t) \equiv$ constant. The same argument holds for any $0<\frac{\varepsilon}{2}<s<\varepsilon$, say. Therefore $\dot{u}_{i}=P(u, x) \equiv 0$ on an open set and hence $P \equiv 0$ on the given torus, showing that $u_{i}$ is a constant of the motion for that torus. If the divisor $\Gamma$ were invariant under the flow for an open set of tori, $P$ would be identically zero by analyticity, which is a contradiction.

We now show that (1) is a Hamiltonian system. Let

$$
\nabla=\left(\frac{\partial}{\partial x_{1}}, \ldots, \frac{\partial}{\partial x_{l+1}}, \frac{\partial}{\partial u_{1}}, \ldots, \frac{\partial}{\partial u_{l+1}}\right)^{\dagger}
$$

and

$$
\mathscr{E}=\left(\begin{array}{cc}
0 & F \\
-F^{\dagger} & 0
\end{array}\right), F_{i j}=x_{i} e_{i j}, 1 \leqq i, j \leqq l+1
$$

define the Poisson bracket ${ }^{1}$

$$
\begin{aligned}
\left\{H, H^{\prime}\right\} & \equiv\left\langle\mathscr{E} \nabla H, \nabla H^{\prime}\right\rangle=\sum_{i, j} F_{i j}\left(\frac{\partial H}{\partial u_{j}} \frac{\partial H^{\prime}}{\partial x_{i}}-\frac{\partial H}{\partial x_{i}} \frac{\partial H^{\prime}}{\partial u_{j}}\right) \\
& =\sum_{i, j} e_{i j}\left(\frac{\partial H}{\partial u_{j}} \frac{\partial H^{\prime}}{\partial v_{i}}-\frac{\partial H}{\partial v_{i}} \frac{\partial H^{\prime}}{\partial u_{j}}\right)
\end{aligned}
$$

$1\langle$,$\rangle denotes the customary inner product$ 
where $v_{i}=\ln x_{i}$. Note in $(u, v)$ coordinates, the Poisson bracket is defined as above except that in $\mathscr{E}, F$ is replaced by $E$, turning the transformed $\mathscr{E}$ into a skewsymmetric constant matrix. But such a matrix always defines a bracket which satisfies the Jacobi identity, as the reader may easily check for himself using the symmetry of the matrix $\nabla(\nabla H)$. Having proved the Jacobi identity in one set of coordinates, we have proved it in any set of coordinates.

Observe that for $H^{\prime}=x_{i}$ and $H^{\prime}=u_{i}$,

$$
\dot{x}_{i}=\left\{H, x_{i}\right\}=x_{i} \sum_{j} e_{i j} \frac{\partial H}{\partial u_{j}}
$$

and

$$
\dot{u}_{i}=\left\{H, u_{i}\right\}=-\sum_{j} x_{j} e_{j i} \frac{\partial H}{\partial x_{j}},
$$

which for

$$
H=\frac{1}{2} \sum_{1}^{l+1} u_{i}^{2}-\sum_{1}^{l+1} x_{i}
$$

amounts to (1).

Let $e_{i}=\left(e_{i 1}, \ldots, e_{i, l+1}\right), 1 \leqq i \leqq l+1$. The matrix $E=\left(e_{i j}\right)$ is assumed to be of rank $l$, so that for some $p$ and $\alpha \neq 0, E \alpha=0$ and $E^{\dagger} p=0$; hence

$$
\begin{gathered}
\sum_{1}^{l+1} p_{i} e_{i}=0, \\
\left\langle e_{i}, \alpha\right\rangle=0, \quad 1 \leqq i \leqq l+1 .
\end{gathered}
$$

The expressions

$$
H_{0} \equiv \sum_{1}^{l+1} x_{i}^{p_{l}} \text { and } \quad H_{1} \equiv \sum_{1}^{l+1} \alpha_{i} u_{i}
$$

are invariants, because

$$
\dot{H}_{0}=\sum_{j} p_{j} \prod_{i \neq j} x_{i}^{p_{i}} x_{j}^{p_{j}-1} \dot{x}_{j}=\prod_{i} x_{i}^{p_{2}} \sum_{k} u_{k} \sum_{j} p_{j} e_{j k}=0
$$

and

$$
\dot{H}_{1}=\sum_{1} \alpha_{i} \dot{u}_{i}=\sum_{i} \alpha_{i} \sum_{j} x_{j} e_{j i}=\sum_{j} x_{j} \sum_{i} \alpha_{i} e_{j i}=0 .
$$

Moreover, both expressions generate zero vector fields, as follows from substituting $H=H_{0}$ or $H_{1}$ into (2). The reader may wonder why we impose a twodimensional degeneracy of the Poisson bracket yielding the two trivial invariants $H_{0}$ and $H_{1}$. From experience, algebraically integrable systems always seem to come equipped with trivial invariants. The reason is unknown, but may be related to the problem of embedding families of affine parts of algebraic tori into $\mathbb{C}^{k}$.

For future notation, let $u=\left(u_{1}, \ldots, u_{l+1}\right)$ and $x=\left(x_{1}, \ldots, x_{l+1}\right)$; let $\tilde{e}_{i j}$ be zero if $e_{i j}=0$, and 1 otherwise. 
Theorem 1. Consider the Hamiltonian system

$$
\begin{gathered}
\dot{x}_{i}=x_{i}\left\langle e_{i}, u\right\rangle, \quad 1 \leqq i \leqq l+1, \\
\dot{u}=\sum_{1}^{l+1} x_{j} e_{j},
\end{gathered}
$$

defined by the system of dependent vectors $e_{i} \in \mathbb{R}^{l+1}$ with the property that every proper subsystem is independent:

$$
\sum_{1}^{l+1} p_{j} e_{j}=0, \text { all } p_{j} \neq 0
$$

It is also assumed that

$$
\sum p_{j} \neq 0 \text {. }
$$

This Hamiltonian system is algebraically completely integrable with abelian functions $u_{i}$ and $x_{i}$, if and only if

$$
A=\left(a_{i j}\right) \equiv\left(\frac{2\left\langle e_{i}, e_{j}\right\rangle}{\left\|e_{j}\right\|^{2}}\right)
$$

is one of the matrices listed in Appendix 1 ; each such matrix is the Cartan matrix of a Kac-Moody Lie algebra. ${ }^{2}$ Then the system linearizes on some abelian variety; if $\theta_{i}(1 \leqq i \leqq l+1)$ denotes a specific translate of the $\theta$-divisor on the abelian variety, then the divisor structure of the $x_{i}$ and $u_{i}$ is as follows:

$$
\left(x_{i}\right)=-\sum_{j=1}^{l+1} a_{i j} \theta_{j}+a \text { positive divisor distinct from the } \theta_{i}{ }^{\prime} s,
$$

and

$$
\left(u_{i}\right)=-\sum \tilde{e}_{j i} \theta_{j}+a \text { positive divisor distinct from the } \theta_{i} \text { 's }
$$

Remark 2. As a consequence of Theorem 1, we may take all $p_{j}>0$; this follows from the Frobenius-Perron theorem applied to the matrix $M \equiv E E^{\dagger}=\left\{\left\langle e_{i}, e_{j}\right\rangle\right\}$. For then $M$ is a positive matrix such that $M_{i j} \leqq 0(i \neq j)$ with $\operatorname{det} M=0$, and so it has a unique null vector $p$ with entries $p_{j}$ all of the same sign; see Carter [3, p. 162].

Remark 3. If we assume the $e_{i}$ 's independent, then by a simpler version of the proof below, algebraic complete integrability occurs if and only if $A$ is the Cartan matrix of a finite dimensional simple algebra. In this case, the algebraic torus should be interpreted as a generalized abelian variety, with infinite periods (see McKean [9]).

Remark 4. The equations above can also be obtained from the Hamiltonian system $\dot{x}_{i}=\frac{\partial H}{\partial y_{i}}(x, y), \dot{y}_{i}=-\frac{\partial H}{\partial x_{i}}, i=1, \ldots, l$, where

$$
H=\frac{1}{2} \sum_{i=1}^{l} y_{1}^{2}+\sum_{i=1}^{l+1} \exp \left\{\sum_{j=1}^{l} N_{i j} x_{j}\right\},
$$

2 See also Helgason [4, p. 503] or Appendix 1, for a very succinct description 
where $N=\left(N_{i j}\right)$ is a full rank matrix of size $(l+1, l)$ whose transpose has a null vector of the form $(\bar{p}, 1)^{\dagger}=\left(p_{1}, \ldots, p_{l}, 1\right)^{\dagger}$; the system is a natural generalization of the Toda equations (see [1]), allowing non-nearest neighbor interactions. The linear transformation preserving $\langle x, y\rangle=\sum_{1}^{l} x_{i} y_{i}$, given by

turns $H$ into

$$
(\bar{x}, \bar{y})=\left(\bar{N} x,\left(\bar{N}^{\dagger}\right)^{-1} y\right), N=\left(\begin{array}{c}
\bar{N} \\
\text { last row }
\end{array}\right)
$$

$$
H=\frac{1}{2}\langle\bar{M} \bar{y}, \bar{y}\rangle+\sum_{i=1}^{l} e^{\bar{x}_{i}}+e^{-\langle\bar{p}, \bar{x}\rangle},
$$

with

$$
\bar{M}=\bar{N} \bar{N}^{\dagger}, N^{\dagger}(\bar{p}, 1)^{\dagger}=0, \bar{p}=\left(p_{1}, \ldots, p_{l}\right)^{\dagger} .
$$

Then, upon using the new variables $a_{i}$ and $b_{i}$ defined by

$$
a=\left(a_{1}, \ldots, a_{l}\right)^{\dagger}=\left(e^{\bar{x}_{1}}, \ldots, e^{\bar{x}_{l}}\right)^{\dagger}, a_{l+1}=e^{-\langle\bar{p}, \bar{x}\rangle}, b=\bar{y},
$$

the Hamilton equations $\dot{\bar{x}}_{i}=\frac{\partial H}{\partial \bar{y}_{i}}, \dot{\bar{y}}_{i}=-\frac{\partial H}{\partial \bar{x}_{i}}, i=1, \ldots, l$, take the form

$$
\begin{aligned}
& \dot{a}_{i}=a_{i} \bar{M}(b)_{i}, i=1, \ldots, l, \dot{b}=-a+\bar{p} a_{l+1} \\
& \dot{a}_{l+1}=-a_{l+1}\langle\bar{M}(b), \bar{p}\rangle .
\end{aligned}
$$

Clearly these equations are completely parametrized by the positive matrix

$$
M=\left[\begin{array}{cc}
\bar{M} & -\bar{M} \bar{p} \\
-(\bar{M} \bar{p})^{\dagger} & \langle\bar{M} \bar{p}, \bar{p}\rangle
\end{array}\right]=N N^{\dagger} \text { with null vector }(\bar{p}, 1)^{\dagger} ;
$$

observing that $\left(\prod_{1}^{l} a_{i}^{p_{2}}\right) a_{l+1}$ is a constant of the motion, it is natural to impose the condition that all $p_{i} \neq 0$. It is easy to check that Eq. (1) is equivalent to (1'), with

$$
M=E E^{\dagger}, a_{i}=x_{i}, E^{\dagger}\left(\begin{array}{l}
b \\
0
\end{array}\right)=u, H_{1}=\langle\alpha, u\rangle=0,
$$

the last condition, $H_{1}=0$, being imposed for the solvability of the previous condition. One may view $\left(1^{\prime}\right)$ as a nonsymmetric normal form for (1). From this discussion the integrability condition depends on $E$ through the matrix $M$ only; in fact $2 M_{i j} / M_{j j}=a_{i j}$ is the Cartan matrix.

Proof. To begin with, observe that if $x_{i}$ has a pole, then $\dot{x}_{i} / x_{i}=\sum_{j} e_{i j} u_{j}$ has a simple pole and therefore also some of the $u_{j}$. Conversely, if $u_{i}$ has a pole, then $\dot{u}_{i}=\sum_{j} x_{j} e_{j i}$ has a pole and therefore also some of the $x_{j}$. Let the vectors $x$ and $u$ have the following asymptotic expansions

$$
x=\frac{x^{(m)}}{t^{m}}+\ldots \text { and } u=\frac{u^{(k)}}{t^{k}}+\ldots \text { with } x^{(m)} \text { and } u^{(k)} \neq 0 .
$$


Then from (1)

$$
-m \frac{x_{i}^{(m)}}{t^{m+1}}+\ldots=\dot{x}_{i}=\left(\frac{x_{i}^{(m)}}{t^{m+k}}+\ldots\right)\left(\left(E u^{(k)}\right)_{i}+\ldots\right)
$$

and

$$
-k \frac{u^{(k)}}{t^{k+1}}+\ldots=\dot{u}=\frac{E^{\dagger} x^{(m)}}{t^{m}}+\ldots+\frac{E^{\dagger} x^{(k+1)}}{t^{k+1}}+\ldots
$$

Note that $m>k+1$; otherwise $u^{(k)}=0$. We show $k=1$; if not, assume $k>1$. Suppose first that $m>k+1$. Then (4) implies $E^{\dagger}\left(x^{(m)}\right)=0$ and hence $x^{(m)}=c p$, with $c \neq 0$; so for all $i, x_{i}^{(m)} \neq 0$. But then (3) implies, since $k>1$, that $\left(E u^{(k)}\right)_{i}=0$, for all $i$; hence $E u^{(k)}=0$, and so $u^{(k)}=c^{\prime} \alpha, \quad c^{\prime} \neq 0$. Finally (4) implies $E^{\dagger} x^{(k+1)}=-k u^{(k)}=-k c^{\prime} \alpha$, but since for any $z,\left\langle E^{\dagger} z, \alpha\right\rangle=\langle z, E \alpha\rangle=0$, we conclude $-k c^{\prime}\langle\alpha, \alpha\rangle=0$, which is a contradiction. So if $k>1$, we must have $m=k+1$ and again by (4), $-k u^{(k)}=E^{\dagger} x^{(m)}$. But since $k>1$, (3) implies ${ }^{3}$

$$
0=\sum x_{i}^{(m)}\left(E \bar{u}^{(k)}\right)_{i}=\left\langle x^{(m)}, E \bar{u}^{(k)}\right\rangle=\left\langle E^{\dagger} x^{(m)}, \bar{u}^{(k)}\right\rangle=-k\left\langle u^{(k)}, \bar{u}^{(k)}\right\rangle=0,
$$

which is a contradiction; hence $k=1$.

We now show $m=2$. Since from (4), $m \geqq k+1=2$; let us assume $m>2$. Then (4) implies $E^{\dagger} x^{(m)}=0$, so $x^{(m)}=c p, c \neq 0$. But then all $x_{i}^{(m)} \neq 0$, and so (3) implies $\left(E u^{(k)}\right)_{i}=-m$ for all $i$, i.e., $E u^{(k)}=-m \delta$, with $\delta=(1,1, \ldots, 1)^{\dagger}$. Equation (4) implies $-k u^{(k)}=E^{\dagger} x^{(k+1)}$, and so $m k \delta=-k E u^{(k)}=E E^{\dagger} x^{(k+1)}$; since the range of $E E^{\dagger}$ is perpendicular to its null vector $p$, we have a contradiction $0=\langle\delta, p\rangle=\sum p_{i}$; thus $m=2, k=1$.

For two column vectors $z$ and $y \in C^{l+1}$, define

$$
z \cdot y=\left(z_{1} y_{1}, z_{2} y_{2}, \ldots, z_{l+1} y_{l+1}\right)^{\dagger} \text {. }
$$

With this notation Eqs. (1) can be rewritten

$$
\dot{x}=x \cdot E u, \dot{u}=E^{\dagger} x .
$$

Substitute now the following expansions for $x$ and $u$

$$
x=t^{-2}\left(x^{0}+x^{1} t+\ldots\right) \text { and } u=t^{-1}\left(u^{0}+u^{1} t+\ldots\right),
$$

with $x^{0}=\left(x_{i}^{0}\right) \neq 0$ and $u^{0}=\left(u_{i}^{0}\right) \neq 0$, into (1), yielding

$$
\begin{aligned}
& -2 x^{0} t^{-3}+\ldots+(k+1) x^{k+3} t^{k}+\ldots \\
& =\left(x^{0} t^{-2}+\ldots+x^{m+2} t^{m}+\ldots\right) \cdot\left(E u^{0} t^{-1}+\ldots+E u^{n+1} t^{n}+\ldots\right),
\end{aligned}
$$

and

$$
\begin{gathered}
-u^{0} t^{-2}+\ldots+(k+1) u^{k+2} t^{k}+\ldots \\
=E^{\dagger} x^{0} t^{-2}+\ldots+E^{\dagger} x^{k+2} t^{k}+\ldots
\end{gathered}
$$

3 Here, and only here, "-" shall denote complex conjugation 
Identifying the coefficients of the minimal power of $t$ leads to two equations and as a consequence a third one:

$$
\begin{aligned}
x^{0} \cdot\left(E u^{0}+2 \delta\right) & =0, \\
E^{\dagger} x^{0}+u^{0} & =0, \\
x^{0} \cdot\left(E E^{\dagger} x^{0}-2 \delta\right) & =0 .
\end{aligned}
$$

Identifying the coefficients of the next power leads to

$$
\begin{aligned}
& x^{1} \cdot\left(E u^{0}+\delta\right)+x^{0} \cdot E u^{1}=0, \\
& E^{\dagger} x^{1}=0,
\end{aligned}
$$

and in general $^{4}$ for $k \geqq 0$

$$
\begin{aligned}
x^{k} \cdot\left(E u^{0}-(k-2) \delta\right)+x^{0} \cdot E u^{k} & =\Gamma_{k-1} \equiv-\sum_{j=1}^{k-1} x^{j} \cdot E u^{k-j}, \\
(k-1) u^{k} & =E^{\dagger} x^{k}
\end{aligned}
$$

and so combining expressions for $k \geqq 2$ we find

$$
\left\{\begin{array}{l}
x^{0} \cdot E E^{\dagger} x^{k}+(k-1) x^{k} \cdot E u^{0}-(k-1)(k-2) x^{k}=(k-1) \Gamma_{k-1} \\
(k-1) u^{k}=E^{\dagger} x^{k}
\end{array}\right\}
$$

If for all $i, x_{i}^{0} \neq 0$, then by (6), $E u^{0}=-2 \delta$, and so

$$
-2 \sum p_{i}=-2\langle\delta, p\rangle=\left\langle E u^{0}, p\right\rangle=\left\langle u^{0}, E^{\dagger} p\right\rangle=0,
$$

which is a contradiction. Let (after possibly relabeling) $x_{1}^{0}, \ldots, x_{s}^{0} \neq 0$, $x_{s+1}^{0}, \ldots, x_{l+1}^{0}=0,1 \leqq s \leqq l$. For any vector $z$, introduce the notation $\bar{z}=\left(z_{1}, \ldots, z_{s}\right)$, $\underline{z}=\left(z_{s+1}, \ldots, z_{l+1}\right)$, and for any matrix $B$, let $\bar{B}$ be the upper-left $s$ by $s$ minor. With this notation and after some manipulation, Eqs. (6), $\left(6^{\prime}\right)$, and $\left(6^{\prime \prime}\right)$ become:

$$
\begin{aligned}
& \left\{\begin{array}{l}
\text { a) }\left(\overline{E u^{0}}\right)+2 \bar{\delta}=0, \\
\text { b) } E^{\dagger} x^{0}+u^{0}=0, \\
\text { c) }\left(\overline{E E^{\dagger}}\right) \bar{x}_{0}=2 \bar{\delta}, \underline{x}^{0}=0,
\end{array}\right\} \\
& \left\{\begin{array}{l}
\text { a) }-\bar{x}^{1}+\bar{x}^{0} \cdot\left(\overline{E u^{1}}\right)=0, \\
\text { b) } x^{1} \cdot\left(\left(\underline{E u^{0}}\right)+\underline{\delta}\right)=0, \\
\text { c) } E^{\dagger} x^{1}=0,
\end{array}\right\}
\end{aligned}
$$

$\left\{\begin{array}{l}\text { a) } \bar{x}^{0} \cdot\left(\overline{E E^{\dagger} x^{k}}\right)-k(k-1) \bar{x}^{k}=(k-1) \bar{\Gamma}_{k-1}, k \geqq 2, \\ \text { b) } \underline{x}^{k} \cdot\left(\underline{E u_{0}}\right)-(k-2) \underline{x}^{k}=\underline{\Gamma_{k-1}}=-\sum_{j=1}^{k-1} \underline{x}^{j} \cdot\left(\underline{E u^{k-j}}\right), \\ \text { c) }(k-1) u^{k}=E^{\dagger} x^{k} .\end{array}\right\}$

4 The case $k=0$ is to be properly interpreted 
Since any proper subset of the $e_{i}$ 's are independent, $\left(\overline{E E^{\dagger}}\right)=\left\{\left\langle e_{i}, e_{j}\right\rangle\right\}_{1 \leqq i, j \leqq s}$ is an invertible matrix, and so (7) defines $x^{0}, u^{0}$ uniquely, by first determining $x^{0}$ from (7c), and then $u^{0}$ from (7b). Equation $\left(7^{\prime} \mathrm{c}\right)$ forces $x^{1}=c p$, which breaks up into the cases $c \neq 0, c=0$.

Case I. $c \neq 0$. Observe $\left(7^{\prime}\right.$ a) implies $\left(\overline{E u^{1}}\right)=\left(\bar{x}^{0}\right)^{-1} \cdot \bar{x}^{1}$, yielding $l+1-s$ degrees of freedom in the determination of $u^{1}$, and one degree of freedom $c$ in the determination of $x^{1}=c p$; hence $l+2-s$ degrees of freedom in all. Since $x^{1}=c p$, all $x_{i}^{1} \neq 0$, and so $\left(7^{\prime} \mathrm{b}\right)$ implies $\underline{E u^{0}}=-\underline{\delta}$; hence $\left(7^{\prime \prime} \mathrm{b}\right)$ becomes $\underline{x}^{k}(k-1)=-\Gamma_{k-1}$, which we may solve immediately and substitute into (7"a). This ${ }^{5} \overline{\text { yields }}$ $\left(\bar{x}^{0} \cdot\left(\overline{E E^{\dagger}}\right)-k(k-1)\right) \bar{x}^{k}=(k-1) \bar{\Gamma}_{k-1}^{\prime}$. The expression $\bar{\Gamma}_{k-1}^{\prime}$ depends on $x^{0}, \ldots, x^{k-1}$, $u^{0}, \ldots, u^{k-1}$. Since a degree of freedom appears in the determination of $\bar{x}^{k}$ precisely when $k(k-1)$ is in the spectrum of $\bar{x}^{0} \cdot\left(\overline{E E^{\dagger}}\right)$, we pick up at most $s$ degrees for $k \geqq 2$, and thus at most a total of $(l+2-s)+s=l+2$ degrees of freedom in the power series. But $l+2 \leqq 2 l+1$ and equal only if $l=1$, a case which is automatically integrable.

Case II. $c=0$. Then $x^{1}=0$. Equation ( $7^{\prime}$ a) implies $\left(\overline{E u^{1}}\right)=0$, yielding $l+1-s$ degrees of freedom at $k=1$. For $k \geqq 2$, we first solve the diagonal system $\left(7^{\prime \prime} b\right)$, producing at most $l+1-s$ degrees of freedom for all $k \geqq 2$. After substituting $\underline{x}^{k}$ into $\left(7^{\prime \prime} \mathrm{a}\right)$, we need only solve

$$
\left[\bar{x}^{0} \cdot\left(\overline{E E^{\dagger}}\right)-k(k-1)\right] \bar{x}^{k}=(k-1) \bar{\Gamma}_{k-1}^{\prime}, k \geqq 2,
$$

in $\bar{x}^{k}$, which together with $\underline{x}^{k}$, yields $u^{k}$ by $\left(7^{\prime \prime} \mathrm{c}\right)$. As before, this equation produces at most $s$ degrees of freedom. In total, at most $(l+1-s)+(l+1-s)+s=2 l+2-s \leqq 2 l+1$ degrees of freedom may arise in our power series; so, unless $s=1$, the number of degrees of freedom $<2 l+1$. Incidentally, since $\bar{x}^{0} \cdot\left(\overline{E E^{\dagger}}\right) \bar{x}^{0}=\bar{x}^{0} \cdot(2 \bar{\delta})=2 \bar{x}_{0}$ holds, $k(k-1)=2$ is always an eigenvalue of $x^{0} \cdot\left(\overline{E E^{\dagger}}\right)$, picking up a degree of freedom at the $k=2$ stage. Thus, only in the case $s=1$, it may be possible to find exactly $2 l+1$ degrees of freedom. Equations ( 7$)$ and $\left(7^{\prime}\right)$ can then be rewritten:

$$
\begin{aligned}
x_{1}^{0}\left\|e_{1}\right\|^{2} & =2, \\
x_{j}^{0} & =0,2 \leqq j \leqq l+1, \\
u^{0} & =-x_{1}^{0} e_{1}=-2\left\|e_{1}\right\|^{-2} e_{1},
\end{aligned}
$$

and

$$
\begin{aligned}
x_{j}^{1} & =0,1 \leqq j \leqq l+1, \\
\left\langle e_{1}, u^{1}\right\rangle & =0 .
\end{aligned}
$$

Hence $u^{1}$ is determined up to $l$ degrees of freedom. An additional one comes from the fact that

$$
x_{1}^{0}\left\|e_{1}\right\|^{2}-k(k-1)
$$

$5 \quad\left(\bar{x}^{0} \cdot\left(\overline{E E^{\dagger}}\right)\right) \bar{x}^{k} \equiv \bar{x}^{0} \cdot \overline{E E^{\dagger}} \bar{x}^{k}$ 
vanishes for $k=2$ by $\left(7^{\prime \prime \prime}\right)$. The required degrees of freedom will then exactly be achieved by having [see $\left.\left(7^{\prime \prime} b\right)\right]$

$$
\begin{aligned}
\left(E u_{0}\right)_{j}= & \left\langle e_{j}, u^{0}\right\rangle=k-2 \text { for some integer } k \geqq 2, \\
& \text { for all } 2 \leqq j \leqq l+1
\end{aligned}
$$

or, what is the same, ${ }^{6}$

$$
a_{j 1} \equiv \frac{2\left\langle e_{j}, e_{1}\right\rangle}{\left\|e_{1}\right\|^{2}} \in-\mathbb{Z}^{+} \text {for } \quad 2 \leqq j \leqq l+1,
$$

provided at least the system of equations for $\left(7^{\prime \prime}\right)$ is compatible. But $\left(7^{\prime \prime \prime}\right)$ implies that for a given admissible asymptotics for which $x_{1} \rtimes \infty$, the other variables $x_{i}$ remain finite. Therefore, the same argument must be repeated for each $x_{i} \uparrow \infty$, leading to the same relations as in $\left(7^{\prime \prime \prime}\right)$ with the index 1 replaced by $i$ and thus leading to the general condition

$$
a_{i j}=\frac{2\left\langle e_{i}, e_{j}\right\rangle}{\left\|e_{j}\right\|^{2}} \in-\mathbb{Z}^{+} \quad \text { for } \quad 1 \leqq i, j \leqq l+1, i \neq j .
$$

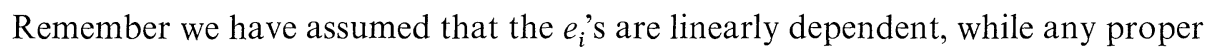
subset of them is independent. Then, according to Helgason [4, pp. 498-503], the matrix $A$ is the Cartan matrix of a Euclidean Lie algebra. ${ }^{7}$ There are only a finite number of them; their Dynkin diagrams are listed in Appendix 1.

Note that by $\left(7^{\prime \prime} \mathrm{b}\right), x_{j}^{k}, k \geqq 2, j \neq i$, stays zero until $k-2=-a_{j i}$, at which point it becomes a free parameter; the usual compatibility conditions are automatically satisfied in this case. Also remember that $x^{1}=0$ and that $x_{i}^{2}$ is free. So summing up, for $x_{i} \rtimes \infty$, we have that

$$
\begin{aligned}
& x_{i}=\frac{2}{\left\|e_{i}\right\|^{2}} t^{-2}+\beta_{i}+\ldots, \\
& x_{j}=\beta_{j} t^{-a_{j i}}+\ldots, j \neq i,
\end{aligned}
$$

with $\beta \in C^{l+1}$ arbitrary and by $(7 b)$, and $\left(7^{\prime}\right)$ with $x^{1}=0$, we find

$$
u=-\frac{2}{\left\|e_{i}\right\|^{2}} e_{i} t^{-1}+\gamma+u^{2} t+\ldots,
$$

where $\gamma \in C^{l+1}$ satisfies $\left\langle\gamma, e_{l}\right\rangle=0$. Then ${ }^{8}$, using $u^{(2)}=E^{\dagger} x^{(2)}$ and $\left(7^{\prime \prime}\right)$,

$$
u^{(2)}=e_{i} \beta_{i}+\sum_{\substack{1 \leqq j \leqq l \\\left\langle e_{j}, e_{i}\right\rangle=0}} e_{j} \beta_{j} .
$$

The vectors, $\beta, \gamma \in C^{l+1}$ with $\left\langle\gamma, e_{i}\right\rangle=0$ account for the $2 l+1$ degrees of freedom. They are in fact the regularizing parameters for the flow (1) near $t=0$.

$6-\mathbb{Z}^{+}=\{0,-1,-2, \ldots\}$

7 See Appendix 1

8 We now use a bracket in $u^{(2)}$, etc., to avoid later confusion 
In Adler and van Moerbeke [2], it was shown that the Hamiltonian systems corresponding to the Kac-Moody Lie algebras are all algebraically completely integrable: they linearize on abelian varieties. Each asymptotic expansion corresponds to some translate of the $\theta$-divisor on this abelian variety; let $\theta_{i}$ be the one on which $x_{i}$ blows up and where the other $x_{j}(j \neq i)$ remain bounded. Then the divisor structure of $x_{j}$ is as follows

$$
\left(x_{j}\right)_{\theta_{i}}=-a_{j i} \theta_{i}
$$

and

$$
\left(u_{j}\right)_{\theta_{i}}=0 \cdot \theta_{i} \text { or }-\theta_{i}
$$

depending on whether $e_{i j}$ is zero or not, hence leading to the conclusions stated in Theorem 1.

The set of vectors $e_{i}$ are determined from the Cartan matrix, up to a common multiplicative constant, and up to a common $l+1$-dimensional rotation, because

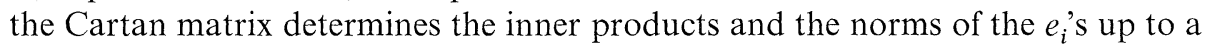
common multiplicative constant. Observe that also the flow induced by Eqs. (1) remains unchanged under a common dilation and under a rotation $O$ of the vectors $e_{i}$; that is to say if $e_{i} \rightarrow O e_{i}$, then $x \rightarrow x, u \rightarrow O u$; indeed $\dot{x}_{i}=x_{i}\left\langle O e_{i}, O u\right\rangle=x_{i}\left\langle e_{i}\right.$, $\left.O^{\dagger} O u\right\rangle=x_{i}\left\langle e_{i}, u\right\rangle, 1 \leqq i \leqq l+1$, and $(O u)=\sum x_{j}\left(O e_{j}\right)$.

In Appendix 1, we exhibit, for each Cartan matrix, a set of root vectors $e_{i}$. As is well known

$$
\sum p_{i} e_{i}=0
$$

can be realized with integers $p_{i}>0$. The $e_{i}$ 's are picked such that the columns of $E$ are the dual root vectors and hence

$$
\left\langle e_{i}, \alpha\right\rangle=0
$$

for integers $\alpha_{i}>0$. These integers lead to the two trivial invariants

$$
H_{0}=\prod_{1}^{l+1} x_{i}^{p_{l}} \text { and } H_{1}=\sum_{1}^{l+1} \alpha_{i} u_{i}
$$

The theta-divisors on each abelian variety can be computed as follows: the systems of differential equations above have besides the two trivial invariants and the energy

$$
H_{0}=\prod_{1}^{l+1} x_{i}^{p_{2}}, \quad H_{1}=\sum_{1}^{l+1} \alpha_{i} u_{i}, \quad H_{2}=\frac{1}{2} \sum_{1}^{l+1}\left(u_{i}^{2}-2 x_{i}\right),
$$

$l-1$ other invariants $H_{i}, 2 \leqq i \leqq l+1$. Along each divisor $\theta_{i}$, each invariant $H_{i}$ is finite and can be expressed after substitution by the expansions $u_{j}$ and $x_{j}$, as a polynomial function in $\beta_{k}, 1 \leqq k \leqq l+1$ and $\gamma_{k}, 1 \leqq k \leqq l+1, k \neq i+1$. This recipe provides $l+2$ equations between these $2 l+1$ parameters, defining an $l$-1-dimensional variety or, what is the same, a codimension 1 subvariety of the abelian variety $\mathscr{A}$ on which the equations linearize. 
Fig. 1

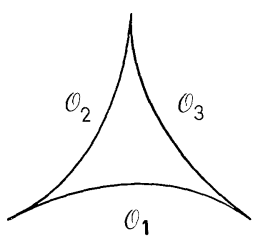

Example. We study the case of the Kac-Moody Lie algebra $a_{2}^{(1)}$. For instance, at the divisor $\mathcal{O}_{1}$, say,

$$
\begin{aligned}
& H_{0}=x_{1} x_{2} x_{3}=\beta_{2} \beta_{3}, \\
& H_{1}=u_{1}+u_{2}+u_{3}=2 \gamma_{1}+\gamma_{3}, \\
& H_{2}=\frac{1}{2}\left(u_{1}^{2}+u_{2}^{2}-2 x_{1}\right)+\frac{1}{2} u_{3}^{2}-x_{2}-x_{3}=\left(\gamma_{1}^{2}-3 \beta_{1}\right)+\gamma_{3}^{2} / 2, \\
& H_{3}=u_{3}\left(u_{1} u_{2}+x_{1}\right)+u_{1} x_{2}+u_{2} x_{3}=\left(3 \beta_{1}+\gamma_{1}^{2}\right) \gamma_{3}+\beta_{2}-\beta_{3} .
\end{aligned}
$$

So, at $\mathcal{O}_{1}$, after elimination of $\gamma_{2}, \gamma_{3}, \beta_{1}$, and $\beta_{3}$, we find that

$$
\beta_{2}-\frac{H_{0}}{\beta_{2}}-P_{3}\left(\gamma_{1}\right)=0
$$

where $P_{3}$ is a cubic polynomial with coefficients depending on $H_{i}(0 \leqq i \leqq 3)$. This equation describes a hyperelliptic curve of genus 2 ; in fact this curve is a copy of the hyperelliptic curve, of which $\mathscr{A}$ is the Jacobi variety. We conclude that for $l=2$, the divisor $\mathscr{D}$ consists of three hyperelliptic curves, pairwise intersecting according to double points (see Fig. 1); indeed one curve $\mathcal{O}_{1}$ has a point in common with another one, only when $\beta_{2}=0$ or $\infty$ and two such curves always intersect doubly.

It will now be shown that the coefficients of the Laurent expansions of $u$ and $x$ actually provide regularizing coordinates of the flow, (1), in a neighborhood of $\mathcal{O}_{1}$. The variables $\gamma$ and $\beta$ parametrize the point of intersection of the trajectory with $\mathcal{O}_{1}$, while $x_{2}$ measures time along the trajectories, and together these data parametrize a neighborhood of $\mathcal{O}_{1}$ in $\mathscr{A}$. To see this, define on the hyperplane $u_{1}+u_{2}+u_{3}=H_{1}$, the following birational map as suggested by the Laurent series for $u, x$ :

$$
\begin{aligned}
&\left(u_{1}, u_{2}, u_{3}, x_{1}, x_{2}, x_{3}\right) \mapsto\left(u_{1} x_{2}, u_{2} x_{3}, u_{3}, x_{1}+u_{1} u_{2}, x_{2}, x_{3}\right) \\
& \equiv\left(\hat{\beta}_{2},-\hat{\beta}_{3}, \hat{\gamma}_{3}, 3 \hat{\beta}_{1}+\hat{\gamma}_{1}^{2}, x_{2}, x_{3}\right) ;
\end{aligned}
$$

putting $2 \hat{\gamma}_{1} \equiv H_{1}-\hat{\gamma}_{3}$, the inverse map reads

$$
\begin{aligned}
& u_{1}=\hat{\beta}_{2} / x_{2}, x_{1}=3 \hat{\beta}_{1}+\hat{\gamma}_{1}^{2}+\hat{\beta}_{2} \hat{\beta}_{3} / x_{2} x_{3}, \\
& u_{2}=-\hat{\beta}_{3} / x_{3}, x_{2}=x_{2}, \\
& u_{3}=\hat{\gamma}_{3}, x_{3}=x_{3} .
\end{aligned}
$$

Now using $\frac{1}{2} H_{1}^{2}-H_{2}=\left(u_{1} u_{2}+x_{1}\right)+u_{3}\left(u_{1}+u_{2}\right)+x_{2}+x_{3}$, in the fourth line below, express the constants of the motion in these new coordinates:

$$
\begin{aligned}
& H_{0}=\left(3 \hat{\beta}_{1}+\hat{\gamma}_{1}^{2}\right) x_{2} x_{3}+\hat{\beta}_{2} \hat{\beta}_{3}, \\
& H_{1}=\left(\hat{\beta}_{2} / x_{2}-\hat{\beta}_{3} / x_{3}\right)+\hat{\gamma}_{3},
\end{aligned}
$$


hence

$$
\begin{aligned}
x_{2} H_{1} & =\hat{\beta}_{2}-\hat{\beta}_{3}\left(x_{2} / x_{3}\right)+\hat{\gamma}_{3} x_{2}, \\
\frac{1}{2} H_{1}^{2}-H_{2} & =3 \hat{\beta}_{1}+\hat{\gamma}_{1}^{2}+\hat{\gamma}_{3}\left(H_{1}-\hat{\gamma}_{3}\right)+x_{2}+x_{3}, \\
H_{3} & =\hat{\gamma}_{3}\left(3 \hat{\beta}_{1}+\hat{\gamma}_{1}^{2}\right)+\hat{\beta}_{2}-\hat{\beta}_{3} .
\end{aligned}
$$

The divisor $\mathcal{O}_{1}$ is given, in the new coordinates, by setting $x_{2}=0$ and $x_{3}=0$ in (10), which leads to the same expression (8) with $\hat{\beta}, \hat{\gamma}$ replacing $\beta, \gamma$, and thus the same variety $\hat{\beta}_{2}-H_{0} / \hat{\beta}_{2}-P_{3}\left(\hat{\gamma}_{3}\right)=0$ and $x_{2}=0$.

We shall use the expression for $H_{1}$ in (10) to show that $x_{2}=0$ forces $x_{3}=0$, and conversely, and also to extend the function $\delta \equiv\left(x_{2} / x_{3}\right)$ to $\mathcal{O}_{1}$. Rewrite the expression for $H_{1}$ as $x_{2} H_{1}=\hat{\beta}_{2}-\hat{\beta}_{3} \delta+\hat{\gamma}_{3} x_{2}$. Upon setting $x_{2}=0$, we find $\hat{\beta}_{2}=\hat{\beta}_{3} \delta$. This equation extends the expression $\delta$ to $\mathcal{O}_{1}$, by defining $\left.\left.(\delta)\right|_{\mathcal{O}_{1}} \equiv\left(\hat{\beta}_{2} / \hat{\beta}_{3}\right)\right|_{\mathcal{O}_{1}}$. Since $x_{3}=\delta^{-1} x_{2}, x_{2}=0$ forces $x_{3}=0$, and conversely; hence in these new coordinates $\mathcal{O}_{1}$ is specified by $x_{2}=0$ or $x_{3}=0$. Since from (9), $u_{2} / u_{1}=-\left(\hat{\beta}_{3} / \hat{\beta}_{2}\right) \delta$, the above expressions continue $u_{1} / u_{2}$ to $\mathcal{O}_{1}$ by the formula $\left.\left(u_{1} / u_{2}\right)\right|_{\mathcal{O}_{1}} \equiv-1$. Besides, in the expression appearing on the third line of $(10),\left(\hat{\beta}_{2} / x_{2}-\hat{\beta}_{3} / x_{3}\right)=\left[\left(1 / x_{2}\right)\left(\hat{\beta}_{2}-\hat{\beta}_{3} \delta\right)\right]$, is identically equal to $H_{1}-\hat{\gamma}_{3} \equiv 2 \hat{\gamma}_{1}$, and so is continued on $\mathcal{O}_{1}$ to equal $2 \hat{\gamma}_{1}$.

In the new coordinates, the differential equation (1)

$$
\begin{array}{ll}
\dot{x}_{1}=x_{1}\left(-u_{1}+u_{2}\right), & \dot{u}_{1}=-x_{1}+x_{3}, \\
\dot{x}_{2}=x_{2}\left(-u_{2}+u_{3}\right), & \dot{u}_{2}=x_{1}-x_{2}, \\
\dot{x}_{3}=x_{3}\left(-u_{3}+u_{1}\right), & \dot{u}_{3}=x_{2}-x_{3},
\end{array}
$$

takes the form:

$$
\begin{aligned}
& \dot{\hat{\beta}}_{2}=\hat{\beta}_{2} \hat{\gamma}_{3}-x_{2}\left(3 \hat{\beta}_{1}+\hat{\gamma}_{1}^{2}\right)+x_{2} x_{3}, \\
& \dot{\hat{\beta}}_{3}=-\hat{\beta}_{3} \hat{\gamma}_{3}-x_{3}\left(3 \hat{\beta}_{1}+\hat{\gamma}_{1}^{2}\right)+x_{2} x_{3}, \\
& \dot{\hat{\gamma}}_{3}=x_{2}-x_{3}, \\
& \dot{\hat{\beta}}_{1}=-\frac{1}{3}\left(\hat{\beta}_{2}+\hat{\beta}_{3}\right)+\frac{1}{6}\left(H_{1}-\hat{\gamma}_{3}\right)\left(x_{2}-x_{3}\right), \\
& \dot{x}_{2}=\left(x_{2} / x_{3}\right) \hat{\beta}_{3}+x_{2} \hat{\gamma}_{3}, \\
& \dot{x}_{3}=\left(x_{3} / x_{2}\right) \hat{\beta}_{2}-x_{3} \hat{\gamma}_{3},
\end{aligned}
$$

which on $\mathcal{O}_{1}\left(x_{2}=x_{3}=0\right)$ defines the vector field

$$
\begin{array}{ll}
\dot{\hat{\beta}}_{2}=\hat{\beta}_{2} \hat{\gamma}_{3}, & \dot{\hat{\beta}}_{1}=-\frac{1}{3}\left(\hat{\beta}_{2}+\hat{\beta}_{3}\right), \\
\dot{\hat{\beta}}_{3}=-\hat{\beta}_{3} \hat{\gamma}_{3}, & \dot{x}_{2}=\hat{\beta}_{2}, \\
\dot{\hat{\gamma}}_{3}=0, & \dot{x}_{3}=\hat{\beta}_{3} .
\end{array}
$$

Since $0 \neq H_{0}=\hat{\beta}_{2} \hat{\beta}_{3}$ on $\mathcal{O}_{1}$, we have $\dot{x}_{2}$ and $\dot{x}_{3} \neq 0$ there. As a result, the flow (11) is transversal to $\mathcal{O}_{1}$, i.e., $\mathcal{O}_{1}$ is a section for the flow and $\hat{Z}=\left(\hat{\beta}_{2}, \hat{\beta}_{3}, \hat{\gamma}_{3}, \hat{\beta}_{1}, x_{2}\right)$ is a set of regularizing variables for the flow near $\mathcal{O}_{1}$. The set of coordinates $Z=\left(\beta_{2}, \beta_{3}, \gamma_{3}\right.$, $\left.\beta_{1}, x_{2}\right)$ as illustrated in Fig. 2 is very close to the coordinates $\hat{Z}$ used previously: $\hat{Z}-Z=O(t)=O\left(x_{2}\right)$, where $O\left(x_{2}\right)$ depends on $Z$.

Each divisor $\mathcal{O}_{2}$ and $\mathcal{O}_{3}$ intersect $\mathcal{O}_{1}$ in exactly one double point, given by $\hat{\beta}_{2}=\infty$ and 0 respectively. This will now be discussed. These two points 


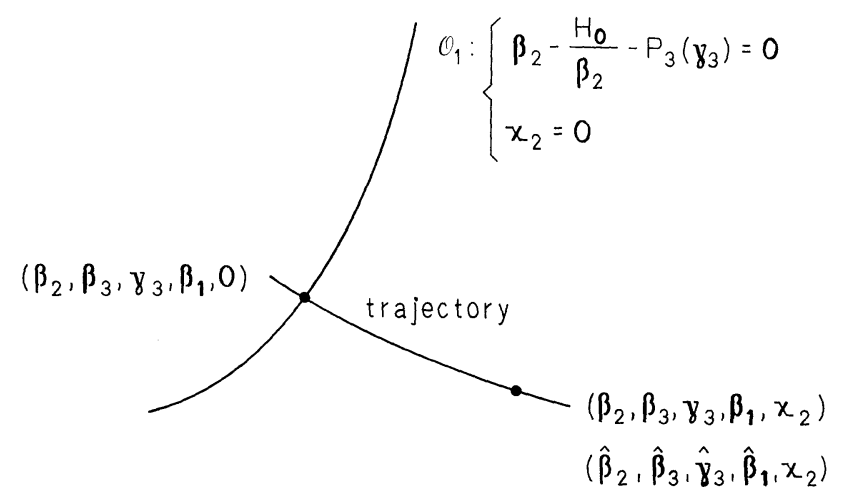

Fig. 2

correspond to the two points at infinity of the curve $\mathcal{O}_{1}$ given by $\hat{\beta}_{2}-H_{0} / \hat{\beta}_{2}-P_{3}\left(\gamma_{1}\right)=0$. That the flow passes through these points can be shown by picking a new set of regularizing coordinates about these points. The flow is regular at $\mathcal{O}_{1} \cap \mathcal{O}_{2}$, and similarly for $\mathcal{O}_{1} \cap \mathcal{O}_{3}$, hence it is regular for $\mathscr{D}=\mathcal{O}_{1} \cup \mathcal{O}_{2} \cup \mathcal{O}_{3}$.

This process of regularization amounts to the compactification of the affine part of $\mathscr{A}$ by glueing on the divisor $\mathscr{D}=\mathscr{O}_{1}+\mathscr{O}_{2}+\mathscr{O}_{1}$; each $\mathscr{O}_{i}$ sits in a coordinate patch whose coordinate functions are ratios from $\mathscr{L}(\mathscr{D})^{9}$; this process can also be interpreted as completing the flow. The functions in $\mathscr{L}(\mathscr{D})$ also serve to embed $\mathscr{A}$ in projective space. By the Riemann-Roch theorem, $\operatorname{dim} \mathscr{L}(\mathscr{D})=9$; below we present a set of functions in $\mathscr{L}(\mathscr{D})$

$$
\begin{gathered}
u_{1} u_{2}+x_{1}, u_{2} u_{3}+x_{2}, u_{3} u_{1}+x_{3}, \\
u_{1}, u_{2}, 1 / x_{1}, 1 / x_{2}, 1 / x_{3}, 1,
\end{gathered}
$$

which suffice to embed $\mathscr{A}$ in projective space.

\section{The Systems of Gross-Neveu}

Recently a new set of Hamiltonian systems have been a subject of interest in particle physics [11]. The equations have the form:

$$
\dot{x}_{j}=\frac{\partial H}{\partial y_{j}}, \dot{y}_{j}=-\frac{\partial H}{\partial x_{j}}, j=1, \ldots, n,
$$

where

$$
H(x, y)=\frac{1}{2} \sum_{j=1}^{n} y_{j}^{2}+\sum_{\alpha \in R} e^{i c\langle\alpha, x\rangle},
$$

with a constant $c, \alpha=\left(\alpha_{1}, \ldots, \alpha_{n}\right),\langle\alpha, x\rangle=\sum_{1}^{n} \alpha_{j} x_{j}$ and $i=\sqrt{-1}$; the sum in $\alpha$ extends over the entire root system $R$ of a simple Lie algebra $L$, as opposed to the

$9 \mathscr{L}(\mathscr{D})$ are the algebraic functions on $\mathscr{A}$ which have at worst a simple pole on $\mathscr{D}$ 
Toda systems, where the sum in $\alpha$ only extends over the simple roots. The case of most immediate physical interest is when $L=o(2 n)$, especially for $n$ small. In this case, the quantum analogue of these systems is thought to be completely integrable when $c=\sqrt{4 \pi}[11]$. Naturally in the classical case, $c$ may be removed from the Hamiltonian by rescaling, and so its value has no bearing on the question of integrability, and we may set $c=1$. It is a natural question to ask whether (1) leads to an algebraically completely-integrable system. Evidence of pair production [in the $o(2 n)$ case] for $c \neq \sqrt{4 \pi}$ indicates the answer is no. We shall apply our criteria to this system for the case $L=\operatorname{sl}(3)$ and show it is not integrable.

Theorem. The Hamiltonian system $\dot{x}_{j}=\frac{\partial H}{\partial x_{j}}, \dot{y}_{j}=-\frac{\partial H}{\partial x_{j}}, j=1,2,3$,

$$
H=\frac{1}{2} \sum_{j=1}^{3} y_{j}^{2}+\sum_{1 \leqq j, k \leqq 3} e^{i\left(x_{j}-x_{k}\right)},
$$

is not algebraically completely integrable, with abelian functions $y_{j}, e^{i x_{j}}, 1 \leqq j \leqq 3$. Hence the Gross-Neveu model for $L=\mathrm{sl}(3)$ is not algebraically integrable.

Proof. For ease of manipulation, we transform the Hamiltonian (1) and hence the Hamilton equations. For later use, the case $s l(l+1)$ will now be considered. Since we shall be working over the complex, we can rescale $(x, y, t=$ time $) \mapsto(i x, y, i t)$ and so $i$ has been removed from (1). As in Sect. 2, we shall transform $(x, y) \mapsto(\tilde{x}, \tilde{y})$ as follows : first set $\tilde{x}_{j}=\left\langle\alpha_{j}, x\right\rangle, j=1, \ldots, l$, for $\left\{\alpha_{j}\right\}_{j=1}^{l}=B$, a simple root system of $R$. Then define $\tilde{x}_{j}=\left\langle\beta_{j}, x\right\rangle, l+1 \leqq j \leqq n$, with the $\beta_{j}$ 's picked so that for all $\alpha_{i}, \beta_{j}$, $\left\langle\alpha_{i}, \beta_{j}\right\rangle=0$, and so $\tilde{x}=A x$ (this defines $A$ ) is an invertible map. Note that every root $\alpha \in R$ is of the form $\alpha= \pm\left(\sum_{j=1}^{l} n_{j} \alpha_{j}\right)$, with $n_{i}=0$ or 1 . Now define $\tilde{y}=\left(A^{T}\right)^{-1} y$, so that $\sum_{1}^{n} x_{j} y_{j}=\sum_{1}^{n} \tilde{x}_{j} \tilde{y}_{j}$; therefore the map $(x, y) \mapsto(\tilde{x}, \tilde{y})$ is canonical. Observe that the Hamiltonian decouples in these coordinates, i.e., it takes the form

$$
\begin{aligned}
H & =H_{0}+H_{1}, H_{0}=\frac{1}{2} \sum_{l+1 \leqq j, k \leqq n}\left\langle\beta_{j}, \beta_{k}\right\rangle \tilde{y}_{j} \tilde{y}_{k}, \\
H_{1} & =\frac{1}{2} \sum_{1 \leqq j, k \leqq l}\left\langle\alpha_{j}, \alpha_{k}\right\rangle \tilde{y}_{j} \tilde{y}_{k}+\sum_{i=1}^{l}\left(e^{\tilde{x}_{2}}+e^{-\tilde{x}_{i}}\right)+\sum_{n \in P}\left(e^{\langle n, \tilde{x}\rangle}-e^{-\langle n, \tilde{x}\rangle}\right),
\end{aligned}
$$

with the sum in $n=\left(n_{1}, \ldots, n_{l}\right)$ extending over the set $P=\left\{n \mid \sum n_{i} \alpha_{i} \in R-B\right.$, all $n_{i}=0$ or 1$\}$. It thus suffices to work with the Hamiltonian $H_{1}$. Now define the $l$ by $l$ matrix $M=\left\{\left\langle\alpha_{j}, \alpha_{k}\right\rangle\right\}$, new coordinates $a_{ \pm i}=e^{ \pm \tilde{x}_{i}}, a_{ \pm n}=e^{ \pm\langle n, x\rangle}, b_{i}=y_{i}, 1 \leqq i \leqq l$, $n \in P$, and the vectors, $a=\left(a_{i}\right), b=\left(b_{i}\right)$; the Hamilton equations $\dot{\tilde{x}}_{i}=\frac{\partial H}{\partial y_{i}}, \dot{\tilde{y}}_{i}=-\frac{\partial H}{\partial x_{i}}$, $i=1, \ldots, l$, expressed in these new coordinates, take the form ${ }^{10}$

$$
\begin{gathered}
\dot{a}=a \cdot M(b), \quad \dot{b}_{i}=a_{-i}-a_{i}+\sum_{n \in P} n_{i}\left(a_{-n}-a_{n}\right), \\
i=1, \ldots, l .
\end{gathered}
$$

10 Given the vectors $x, y$, the symbol $x \cdot y$ denotes a new vector with components $(x \cdot y)_{i}=x_{\imath} y_{\imath}$ 
For the case $L=\operatorname{sl}(3)$, Eqs. (2) take the form

$$
\left\{\begin{array}{l}
\text { (i) }\left(\begin{array}{l}
a_{1} \\
a_{2}
\end{array}\right)^{\cdot}=\left(\begin{array}{l}
a_{1} \\
a_{2}
\end{array}\right) \cdot M\left(\begin{array}{l}
b_{1} \\
b_{2}
\end{array}\right), M=\left[\begin{array}{cc}
2 & -1 \\
-1 & 2
\end{array}\right], \\
\text { (ii) }\left(\begin{array}{l}
b_{1} \\
b_{2}
\end{array}\right)^{\cdot}=\left(\begin{array}{l}
a_{1}^{-1} \\
a_{2}^{-1}
\end{array}\right)-\left(\begin{array}{l}
a_{1} \\
a_{2}
\end{array}\right)+\delta\left(\frac{1}{a_{1} a_{2}}-a_{1} a_{2}\right) ; \delta=\left(\begin{array}{l}
1 \\
1
\end{array}\right) \cdot
\end{array}\right\}
$$

We think of (3) as a differential equation in $C^{4}$; to prove the theorem, it suffices to show that (3) has no 3-parameter pole solutions. First observe that if any of the $a_{i}$ blow up, some of the $b_{j}$ must blow up by Eq. (3i), and conversely by Eq. (3ii). Note $b$ has at most a simple pole, for if not, $b=\frac{b^{0}}{t^{k}}+\ldots, k \geqq 2$, and since $a_{1}$ and $a_{2}$ have the form

$$
a_{1}=\varepsilon_{1} t^{j_{1}}+\ldots, \quad a_{2}=\varepsilon_{2} t^{j_{2}}+\ldots,
$$

(3i) implies

$$
\dot{a}_{1}=\varepsilon_{1} j_{1} t^{j_{1}-1}+\ldots=\left(\varepsilon_{1} t^{j_{1}}+\ldots\right)\left(\frac{\left(M b_{0}\right)_{1}}{t^{k}}+\ldots\right)
$$

hence $\left(M b^{0}\right)_{1}=0$; similarly $\left(M b^{0}\right)_{2}=0$, i.e., $M b^{0}=0$, but since $M$ is a positive definite matrix, $b^{0}=0$.

We also claim that $a_{1}, a_{2}$ cannot blow up simultaneously with three degrees of freedom. For since $\dot{b}$ has at most a double pole, both $a_{1}$ and $a_{2}$ blow up only with simple poles as a consequence of (3ii). So assume

$$
b=\frac{1}{t}\left(b^{0}+b^{1} t+\ldots+b^{j} t^{j}+\ldots\right), \quad a=\frac{1}{t}\left(a^{0}+a^{1} t+\ldots+a^{j} t^{j}+\ldots\right),
$$

with $a^{0}=\left(\begin{array}{l}a_{1}^{0} \\ a_{2}^{0}\end{array}\right)$, and $a_{1}^{0}, a_{2}^{0} \neq 0$. Equating the coefficients of the $t^{-2}$ terms in (3) yields $-a^{0}=a^{0} \cdot M b^{0}$, i.e., $M b^{0}=-\delta$, and $b^{0}=a_{1}^{0} a_{2}^{0}$. Since $M \delta=\delta$ and $b^{0}=-\delta$, we have $a_{1}^{0} a_{2}^{0}=-1$. We next equate coefficients of the $t^{-1}$ terms in (3), concluding

$$
0=a^{0} \cdot M b^{1}+a^{1} \cdot M b^{0}, 0=-a^{0}-\delta\left(a_{1}^{1} a_{2}^{0}+a_{2}^{1} a_{1}^{0}\right) .
$$

The latter equation implies $a^{0}=c \delta$ for some $c$, hence $a_{1}^{0}=a_{2}^{0}$, and so, since $a_{1}^{0} a_{2}^{0}=-1, a^{0}=i \delta$, and by (5), $\left\langle a^{1}, \delta\right\rangle=-1$. Since $M b^{0}=-\delta$ and $a^{0}=i \delta,(5)$ implies $a^{1}=i M b^{1}$, hence $b^{1}$ is determined by $a^{1}$, and so at this point we have one degree of freedom in (4). Substituting (4) into (3), using $b_{0}=-\delta$ and $a_{0}=i \delta$, and upon equating the coefficients of the $t^{j-2}$ terms in (3) for $j \geqq 2$, we find ${ }^{11}$

(i) $(j-1) a^{j}-a_{0} \cdot M b^{j}-a^{j} \cdot M b^{0}=j a^{j}-i M b^{j}=k_{j-1}, j \geqq 2$,

(ii) $(j-1) b^{j}-\delta\left\langle i \delta, a^{j}\right\rangle=(j-1) b^{j}-i \delta \otimes \delta a^{j}=k_{j-1}$,

where $k_{j}$ is a generic symbol for an expression depending on terms with index at most $j$. Substituting (ii) into (i), we find

$$
[M \cdot \delta \otimes \delta+j(j-1)] a^{j}=[\delta \otimes \delta+j(j-1)] a^{j}=k_{j-1}, j \geqq 2 .
$$

$11(v \otimes w)_{i j}=v_{\imath} w_{j}$, hence $v \otimes w(u)=v\langle w, u\rangle$ 
The operator $\delta \otimes \delta$ has spectrum $\{0,2\}$, and so for $j \geqq 2$, there is no degree of freedom in the determination of $a^{j}$ and of $b^{j}$ as well, since from (ii) $b^{j}$ is uniquely determined by $a^{j}$. We thus conclude there exists a power series solution, with precisely one degree of freedom (which enters at $j=1$ ).

If $a_{1}$ had a triple pole, then since $b$ has only a simple pole, and $a_{2}$ cannot blow up, the expression for $\dot{b}_{1}$ in (3) implies $a_{2}$ has a sixth order pole in order to cancel the triple pole of $a_{1}$. But then the expression for $\dot{b}_{2}$ is inconsistent, and in general this argument shows $a_{1}$ has at most a double pole. If, on the contrary, $a_{1}$ has a simple pole, and $a_{2}=c+\mathcal{O}(t)$ or $t(c+\mathcal{O}(t)), c \neq 0$, then since $b_{2}$ has no $1 / t$ terms, the expression (3) for $\dot{b}_{2}$ is inconsistent. Also, since the transformation $a_{i} \rightarrow a_{i}^{-1}$ has only the effect in (3) of reversing time, $t \rightarrow-t$, we need only consider the following three cases

$$
a_{1}=\frac{c_{1}}{t^{2}}+\ldots, \quad a_{2}=\left\{\begin{array}{l}
c_{2} t^{2}+\ldots \\
c_{2} t+\ldots \\
c_{2}+\ldots
\end{array}\right\}, \quad c_{1} c_{2} \neq 0 .
$$

At this point it is convenient to change the variable $a_{2}$ into $-a_{2}^{-1}$, and so (3) becomes

$$
\left\{\begin{array}{l}
\text { (i) }\left(\begin{array}{l}
a_{1} \\
a_{2}
\end{array}\right)^{\cdot}=\left(\begin{array}{l}
a_{1} \\
a_{2}
\end{array}\right) \cdot \bar{M}\left(\begin{array}{l}
b_{1} \\
b_{2}
\end{array}\right), \quad \bar{M}=\left[\begin{array}{ll}
2 & -1 \\
1 & -2
\end{array}\right], \\
\text { (ii) }\left(\begin{array}{l}
b_{1} \\
b_{2}
\end{array}\right)^{\cdot}=\left(\begin{array}{l}
a_{1}^{-1} \\
a_{2}^{-1}
\end{array}\right)-\left(\begin{array}{l}
a_{1} \\
a_{2}
\end{array}\right)+\delta\left(\frac{a_{1}}{a_{2}}-\frac{a_{2}}{a_{1}}\right) .
\end{array}\right\}
$$

We shall deal with all three cases at once. Let

$$
a=\left(\begin{array}{c}
1 / t^{2} \\
t^{2} / t^{2}
\end{array}\right) \cdot\left(a^{0}+a^{1} t+\ldots\right), \quad b=\frac{1}{t}\left(b^{0}+b^{1} t+\ldots\right),
$$

where $\varepsilon=0$ or 1 or 2 ; define $\eta(x)=1$ if $x=0$, and 0 otherwise. Equating the coefficients of order $t^{-3}, t^{-3+\varepsilon}$ in the respective equations (6i) for $\dot{a}_{1}, \dot{a}_{2}$, we find

$$
\bar{M} b^{0}=\left(\begin{array}{c}
-2 \\
\varepsilon-2
\end{array}\right) ;
$$

since $\bar{M}$ is invertible, this uniquely determines $b^{0} \neq 0$. Equating the $t^{-2}$ coefficients in (6ii) yields

$$
-b^{0}=-\left(\begin{array}{c}
a_{1}^{0} \\
a_{2}^{0} \eta(\varepsilon)
\end{array}\right)+\delta\left(a_{1}^{0}\left(a_{2}^{0}\right)^{-1} \eta(\varepsilon-2)\right) .
$$

If $\varepsilon=0,2$, it uniquely determines $a^{0}$ and if $\varepsilon=1$ it determines $a_{1}^{0}=b_{1}^{0}$. To determine $a_{2}^{0}$ in the case $\varepsilon=1$, we need to equate the $t^{-1}$ coefficients in (6ii), concluding

$$
0=-\left(\begin{array}{l}
a_{1}^{1} \\
a_{2}^{0}
\end{array}\right)+\delta\left(a_{1}^{0}\left(a_{2}^{0}\right)^{-1}\right),
$$


and so $a_{2}^{0}=\sqrt{a_{1}^{0}}$. We now substitute (7) into (6i) and equate respectively the $t^{j-3}$, $t^{j-3+\varepsilon}, j \geqq 1$ terms in $\dot{a}_{1}, \dot{a}_{2}$, yielding

$$
\left(\begin{array}{c}
j-2 \\
j-2+\varepsilon
\end{array}\right) \cdot a^{j}-a^{0} \cdot \bar{M} b^{j}-a^{j} \cdot \bar{M} b_{0}=k_{j-1}, \quad j \geqq 1,
$$

and so substituting (8) into the above, we find

$$
j a^{j}-a^{0} \cdot \bar{M} b^{j}=k_{j-1}, \quad j \geqq 1 .
$$

Equating the $t^{j-2}$ coefficients in (6ii) yields

$$
(j-1) b^{j}+\left(\begin{array}{c}
a_{1}^{j} \\
a_{2}^{j} \eta(\varepsilon)
\end{array}\right)-\eta(2-\varepsilon) \delta\left\langle v_{0}, a^{j}\right\rangle=k_{j-1}, \quad j \geqq 1,
$$

with $v_{0}=\left(a_{2}^{0}\right)^{-2}\left(\begin{array}{c}a_{2}^{0} \\ -a_{1}^{0}\end{array}\right)$; we may rewrite the latter as

$$
(j-1) b^{j}-N a^{j}=k_{j-1}, j \geqq 1,
$$

with $N$ determined from the above expression. Substituting (9) into (10) yields

$$
[(j(j-1))-C] b^{j}=k_{j-1}, \quad C=N \cdot a^{0} \cdot \bar{M}, \quad j \geqq 1,
$$

and thus we have at most two degrees of freedom in the determination of $b^{j}, j \geqq 1$. Since by (9), $a^{j}$ is determined uniquely from $b^{j}$ for $j \geqq 1$, we have at most two degrees of freedom in our series solution (7), proving the theorem.

The first case of physical interest is really $L=o(6)=D_{3}$, which however is isomorphic to $L=\operatorname{si}(4)$. We have

Theorem. The Hamiltonian system $\dot{x}_{j}=\frac{\partial H}{\partial y_{j}}, \dot{y}_{j}=-\frac{\partial H}{\partial x_{j}}, j=1,2,3,4$,

$$
H=\frac{1}{2} \sum_{j=1}^{4} y_{j}^{2}+\sum_{1 \leqq j, k \leqq 4} e^{i\left(x_{j}-x_{k}\right)},
$$

is not algebraically completely integrable, with abelian functions $y_{j}, e^{i x_{j}} ; 1 \leqq j \leqq 4$. Hence the Gross-Neveu model for $L=\mathrm{sl}(4) \approx o(6)$ is not algebraically integrable.

Sketch of Proof. Using Eq. (2) for $L=\operatorname{sl}(4)$, the differential equations take the form, with

$$
\begin{aligned}
& a=\left(a_{1}, a_{2}, a_{3}\right)^{\dagger}, b=\left(b_{1}, b_{2}, b_{3}\right)^{\dagger}, \\
& \dot{a}=a \cdot M(b), \quad M=\left[\begin{array}{rrr}
2 & -1 & 0 \\
-1 & 2 & -1 \\
0 & -1 & 2
\end{array}\right], \\
& \dot{b}_{1}=a_{1}^{-1}-a_{1}+e+g, \quad \quad e=a_{1}^{-1} a_{2}^{-1}-a_{1} a_{2}, f=a_{2}^{-1} a_{3}^{-1}-a_{2} a_{3}, \\
& \dot{b}_{2}=a_{2}^{-1}-a_{2}+e+f+g, \quad g=a_{1}^{-1} a_{2}^{-1} a_{3}^{-1}-a_{1} a_{2} a_{3} . \\
& \dot{b}_{3}=a_{3}^{-1}-a_{3}+f+g,
\end{aligned}
$$


Using the fact that (11) possesses the symmetries

$$
\begin{aligned}
\left(b, a_{1}, a_{2}, a_{3}, t\right) & \rightarrow\left(b, a_{1}^{-1}, a_{2}^{-1}, a_{3}^{-1},-t\right), \\
\left(b_{1}, b_{2}, b_{3}, a_{1}, a_{2}, a_{3}\right) & \rightarrow\left(b_{3}, b_{2}, b_{1}, a_{3}, a_{2}, a_{1}\right),
\end{aligned}
$$

an hours reflection shows that the only possible pole solutions of (11), with $c_{1} c_{2} c_{3} \neq 0$, are of the form $b=\frac{1}{t}\left(b^{0}+b^{1} t+\ldots\right)$, and

(i) $a_{1}=\frac{c_{1}}{t}+\ldots, \quad a_{2}=c_{2}+\ldots, \quad a_{3}=\frac{c_{3}}{t}+\ldots$,

(ii) $a_{1}=\frac{c_{1}}{t}+\ldots, \quad a_{2}=\frac{c_{2}}{t}+\ldots, \quad a_{3}=c_{3}+\ldots$,

(iii) $a_{1}=\frac{c_{1}}{t}+\ldots, \quad a_{2}=t^{\varepsilon+1}\left(c_{2}+\ldots\right), \quad a_{3}=\frac{c_{3}}{t}+\ldots$,

$$
\varepsilon=0 \text { or } 1 \text {, }
$$

(iv) $a_{1}=\frac{c_{1}}{t^{2}}+\ldots, \quad a_{2}=t^{\varepsilon+1}\left(c_{2}+\ldots\right), \quad a_{3}=\frac{c_{3}}{t}+\ldots$,

$$
\varepsilon=0 \text { or } 1 \text {, }
$$

(v) $a_{1}=\frac{c_{1}}{t^{2}}+\ldots, \quad a_{2}=c_{2} t^{2}+\ldots, \quad a_{3}=\frac{c_{3}}{t^{2}}+\ldots$,

(vi) $a_{1}=\frac{c_{1}}{t^{k}}+\ldots, \quad a_{2}=c_{2} t^{k}+\ldots, \quad a_{3}=\frac{c_{3}}{t^{k}}+\ldots, \quad k>2$,

(vii) $a_{1}=\frac{c_{1}}{t}+\ldots, \quad a_{2}=\frac{c_{2}}{t}+\ldots, \quad a_{3}=t^{\varepsilon}\left(c_{1}+\ldots\right)$,

$$
\varepsilon=0 \text { or } 1 \text { or } 2 \text {, }
$$

(viii) $a_{1}=\frac{c_{1}}{t^{1+\varepsilon_{1}}}+\ldots, \quad a_{2}=t^{\varepsilon_{2}}\left(c_{1}+\ldots\right), \quad a_{3}=c_{3}+\ldots$,

$$
\varepsilon_{1}=0 \text { or } 1, \varepsilon_{2}=0 \text { or } 1 \text { or } 2 \text {, }
$$

(ix) $a_{1}=\frac{c_{1}}{t^{1+\varepsilon_{1}}}+\ldots, \quad a_{2}=c_{2}+\ldots, \quad a_{3}=t^{1+\varepsilon_{2}}\left(c_{3}+\ldots\right)$,

$$
\varepsilon_{1}, \varepsilon_{2}=0 \text { or } 1 \text {. }
$$

Except for (vi), we now analyze these cases exactly as in the last example. It turns out that some of the cases cannot occur because of a contradiction in the equations for the first terms $b^{0}, a^{0}$. The remaining cases do not permit five parameter solutions. We thus only need analyze case (vi). Equating the coefficients of the highest terms, in the equations $a_{i}=a_{i}(M(b))_{i}, i=1,2,3$, respectively, implies $M\left(b^{0}\right)=\left(\begin{array}{r}-k \\ k \\ -k\end{array}\right), \quad$ hence $b^{0}=-(k / 2)\left(\begin{array}{l}1 \\ 0 \\ 1\end{array}\right)$. 
Thus $b_{2}$ doesn't blow up in this case, nor in any symmetric reflection of this case, which concludes the proof of the theorem.

Remark. Case (vi) still bears further scrutiny. In fact, it is easy to see that one has genuine $k-2$ parameter formal solutions of the form (vi); however, as (11) is a cubic equation, they need not converge, indeed, for $k \geqq 8$, they certainly cannot converge as the formal solution genuinely depends on all the $k-2$ parameters. One suspects that the solutions don't converge for any $k$, but for $k=7$ they lead to a pair of five parameter formal solutions in which only $b_{2}$ has no formal pole. This implies that $b_{2}$ could never be an algebraic function, a fact which we used above. This example illustrates the difference between quadratic and higher order equations, and further suggests their preferential role in this theory.

Acknowledgements. We wish to thank Michael Artin and R. Shankar for many fruitful conversations.

\section{References}

1. Adler, M., van Moerbeke, P.: Completely integrable systems, Euclidean Lie algebras, and curves. Adv. Math. 38, 267-317 (1980)

2. Adler, M., van Moerbeke, P.: Linearization of Hamiltonian systems, Jacobi varieties and representation theory. Adv. Math. 38, 318-379 (1980)

3. Carter, R.W.: Simple groups of Lie type. London, New York: Wiley 1972

4. Helgason, S.: Differential geometry, Lie groups and symmetric spaces. New York: Academic Press 1978

5. Hille, E.: Ordinary differential equations in the complex domain. New York: Wiley-Interscience 1976

6. Humphreys, J.E.: Introduction to Lie algebras and representation theory. Berlin, Heidelberg, New York: Springer 1972

7. Kowalewski, S.: Sur le probleme de la rotation d'un corps solide autour d'un point fixe. Acta Math. 12, 177-232 (1889)

8. Kowalewski, S. : Sur une propriété du système d'équations différentielles qui définit la rotation d'un corps solide autour d'un point fixe. Acta Math. 14, 81-93 (1891)

9. McKean, H.P.: Theta functions, solitons and singular curves, partial differential equations and geometry, pp. 237-254. New York: M. Dekker, Inc. 1979

10. Bogoyavlensky, O.I.: On perturbations of the periodic Toda lattices. Commun. Math. Phys. 51, 201-209 (1976)

11. Shankar, R.: A model that acquires integrability and $O(2 N)$ invariance at a critical coupling. Preprint Yale University YTP 81-06

12. Adler, M., van Moerbeke, P.: The algebraic integrability of geodesic flow on SO(4). Invent. Math. (to appear) (1982)

Communicated by J. Moser

Received August 12, 1981

\section{Appendix 1}

The Cartan matrices above correspond to so-called Kac-Moody Lie algebras. The reader will find information about them in Helgason [4, pp. 490-515]. We sketch a few of the ideas here. Consider a simple Lie algebra $g$ over $\mathbb{C}$ and let $\sigma$ be an outer automorphism of $g$ satisfying $\sigma^{m}=$ identity. Then each eigenvalue of $\sigma$ has the form $\varepsilon^{i}(i, \bmod m)$, where $\varepsilon$ is a primitive root of unity. Let $g_{i}$ be the eigenspace of $\sigma$ for the eigenvalue $\varepsilon^{i}$. Then we have the finite gradation

$$
g=\bigoplus_{0 \leqq i \leqq m} g_{i}
$$


the inclusion $\left[g_{i}, g_{j}\right] \subset g_{i+j}$ holds. The Lie algebra

$$
L(g, \sigma) \equiv \bigoplus_{j \in Z} x^{j} g_{J \bmod m}
$$

is infinite-dimensional. The analogue of the weight theory can be developed for these Kac-Moody Lie algebras. It is shown that $L(g, \sigma)$ is isomorphic to $L(g, v)$, where $v$ is an automorphism induced by an automorphism of the Dynkin diagram of $g$. As is easily seen from inspection, only Dynkin diagrams connected with the algebras $a_{l}, d_{l}$, and $e_{6}$ admit an automorphism of order 2 and the diagram of $d_{4}$ admits an automorphism of order 3 ; the other diagrams have no automorphisms but the identity.

We now explicitly exhibit the Dynkin diagrams, the Cartan matrices, a choice of $E$ and the corresponding $p$.
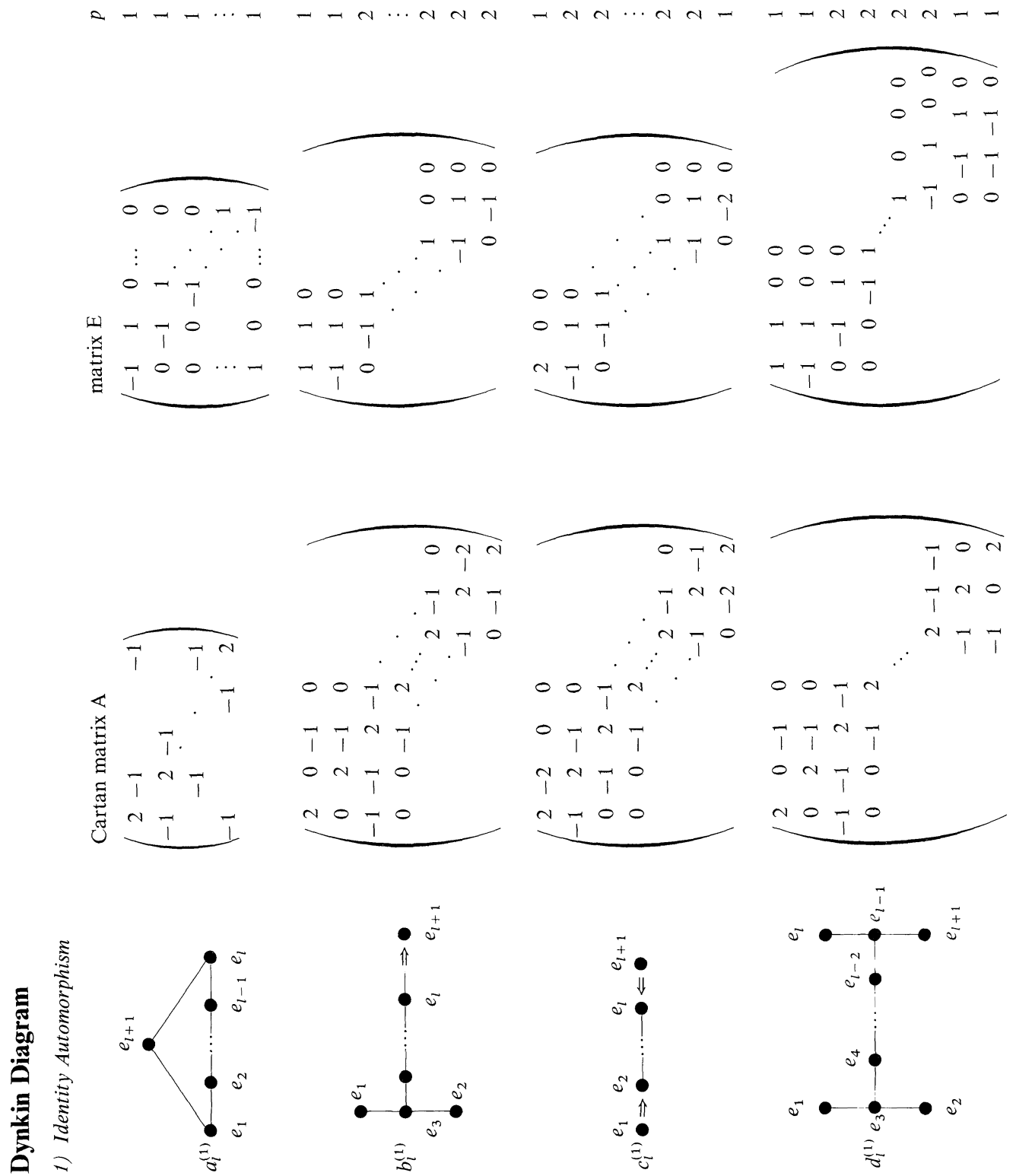


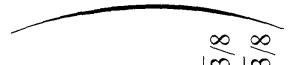

$00000 \stackrel{1}{1}$

$00000 \gg \frac{1}{1}$

$000-\pi \stackrel{1}{\pi}$

$00-\frac{1}{1} \stackrel{\text { N }}{-}$

$0 \underset{+}{-1} 00 \stackrel{\text { N }}{=}$

$-\frac{1}{-1} 000$

- $00000 \stackrel{n}{1}$ $\stackrel{\infty}{\ln 1 m}$

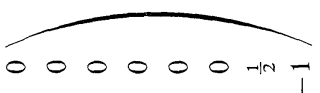

$0.000000 \pi m-1$

$0000-\frac{1}{-N} 0$

$000-\pi-\pi-m o$

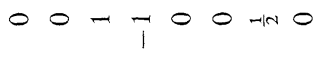

$0-\pi 000-\pi 0$ $-\frac{1}{1} 0000-m 0$
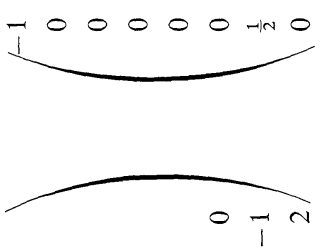

$000-100 \mathrm{~N}$

$0000-100$

$00 \pi 0$ T

$00 \rightarrow N 00 \frac{1}{1}$

$0 \overrightarrow{1} \pi \overrightarrow{1} 00$

$\pi N \vec{r} 0000$

NTO 0000

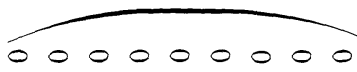

$000000-7-m$

$00000-7-\pi$ -

$0000-700-1 N$

$000-7000-1 N$

$00-\pi 00000 \pi$

$0-T 00000 \pi n$

$-\pi 000000-\pi$

Ho000000 min

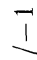

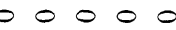

$00-\pi^{-m}$

$0-T 0-1 n$ On

$-700-\pi n-$

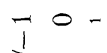

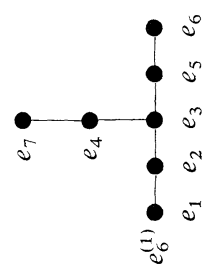
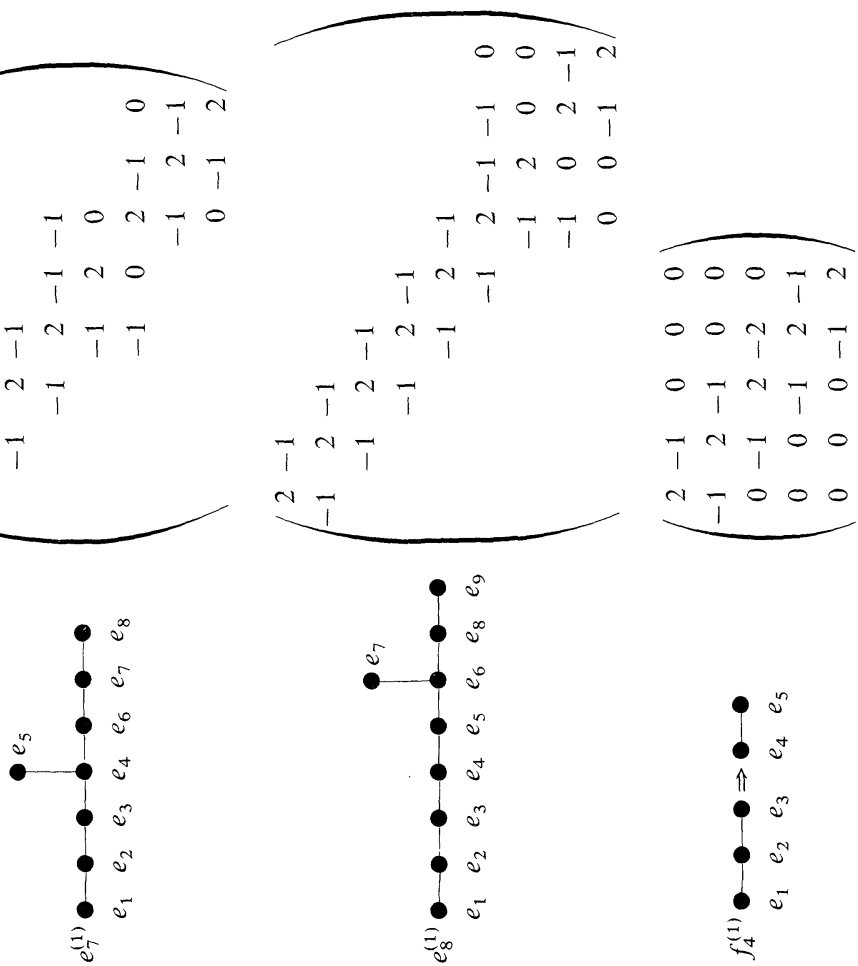

$004 N T$

othto

$\overrightarrow{1} N \vec{T} 00 \quad T$

$\sqrt{\mathrm{N} 000} \frac{\mathrm{n}}{1}$
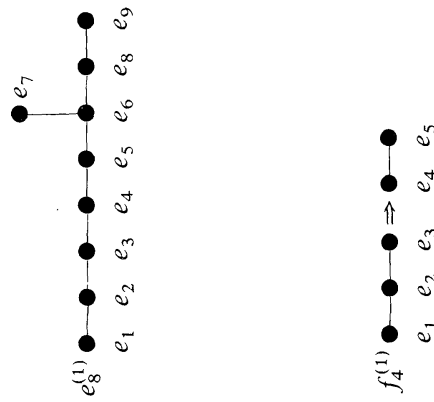
2) Order 2 Automorphisms

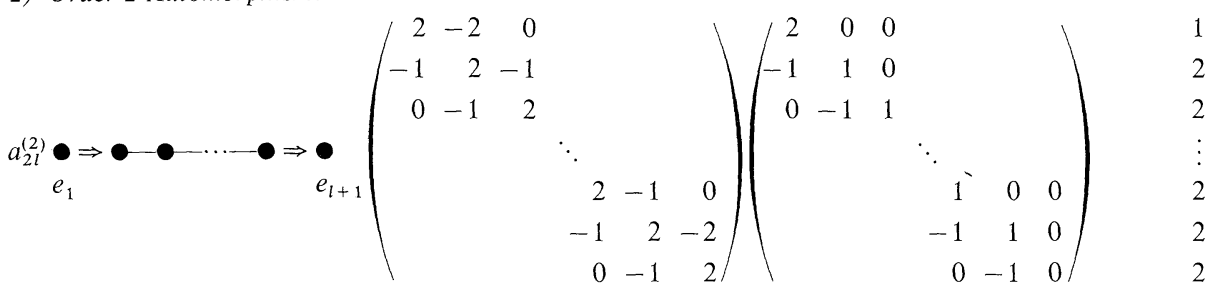

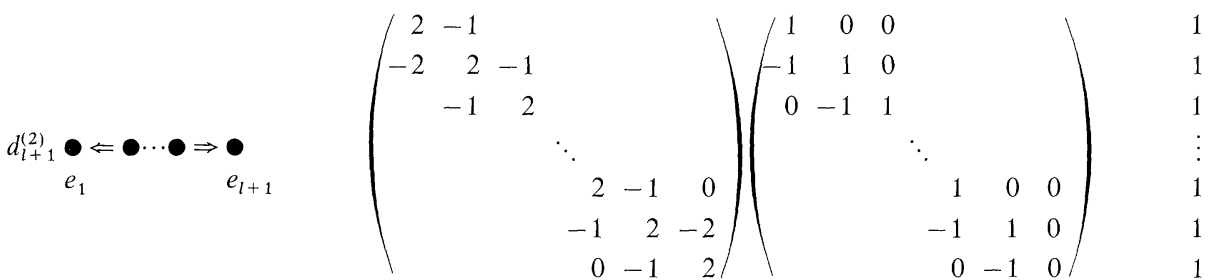

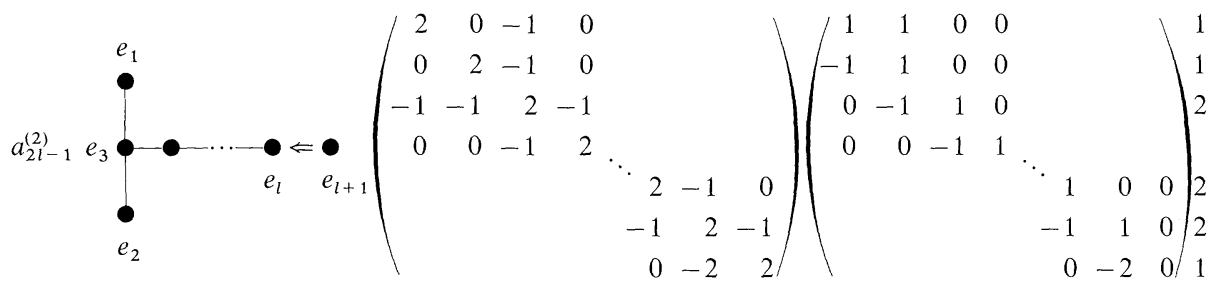

$\left.\begin{array}{lllll}e_{6}^{(2)} & \bullet & \bullet & \bullet & \bullet \\ e_{1} & e_{2} & e_{3} & e_{4} & e_{5} \\ -1 & 2 & -2 & 0 & 0 \\ 0 & -1 & 2 & -1 & 0 \\ 0 & 0 & -1 & 2 & -1 \\ 0 & 0 & 0 & -1 & 2\end{array}\right)\left(\begin{array}{rrrrr}2 & -1 & 0 & 0 & 0 \\ 0 & & & \end{array}\right.$

3) Order 3 Automorphism

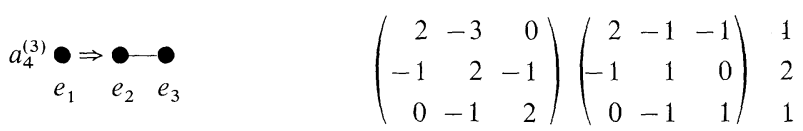

